\title{
Transarterial Onyx Embolization of an Orbital Solitary Fibrous Tumor
}

\author{
Nafiseh Hashemi ${ }^{a}$ Jeanie D. Ling ${ }^{b}$ Charles Soparkar b, c, e, h, k \\ Mirwat Sami $^{e}$ Benjamin Ellezam ${ }^{f}$ Richard Klucznik ${ }^{g}$ \\ Andrew G. Lee ${ }^{a, d}, e, j, k, m$ Patricia Chévez-Barrios $d-f, i, l$ \\ ${ }^{a}$ The University of Texas Health Sciences, Departments of b Plastic Surgery and \\ cOphthalmology at ${ }^{\mathrm{d}}$ Baylor College of Medicine, Departments of e Ophthalmology, \\ ${ }^{\mathrm{f}}$ Pathology and Genomic Medicine and ${ }^{9}$ Radiology, Houston Methodist Hospital, \\ and hivision of Head and Neck Surgery, and 'Pathology and Laboratory Medicine, \\ MD Anderson Cancer Center, University of Texas, Houston Tex., 'j Department of \\ Ophthalmology, The University of Texas Medical Branch, Galveston, Tex., ${ }^{k}$ Department of \\ Ophthalmology, Neurology, and Neurosurgery, and 'Pathology and Laboratory Medicine \\ and Ophthalmology, Weill Cornell Medical College, New York, N.Y., and mepartment of \\ Ophthalmology, The University of Iowa Hospitals and Clinic, Iowa City, Iowa, USA
}

\section{Key Words}

Orbital solitary fibrous tumor · Onyx (ethylene vinyl alcohol copolymer) · Transarterial embolization

\begin{abstract}
Solitary fibrous tumor (SFT) is an uncommon mesenchymal neoplasm sometimes found in the orbit. We report a case of an aggressive orbital SFT with enlarged feeding vessels that was successfully resected immediately after transarterial embolization with Onyx (ethylene vinyl alcohol copolymer). To our knowledge, this is the first report showing the histopathology of Onyx embolization material in an orbital SFT.

(C) 2015 S. Karger AG, Basel
\end{abstract}

\section{Introduction}

Solitary fibrous tumor (SFT) is an uncommon mesenchymal neoplasm sometimes found in the orbit. We report a case of an aggressive orbital SFT with enlarged feeding vessels that was successfully resected immediately after transarterial embolization with Onyx (ethylene vinyl alcohol copolymer). To our knowledge, this is the first report showing the histopathology of Onyx embolization material in an orbital SFT. 


\section{Ocular Oncology} and Pathology

\begin{tabular}{l|l}
\hline Ocul Oncol Pathol 2015;1:98-102 \\
\hline DOI: 10.1159/000370048 & $\begin{array}{l}\text { @ 2015 S. Karger AG, Basel } \\
\text { www.karger.com/oop }\end{array}$ \\
\hline
\end{tabular}

Hashemi et al.: Transarterial Onyx Embolization of an Orbital Solitary Fibrous Tumor

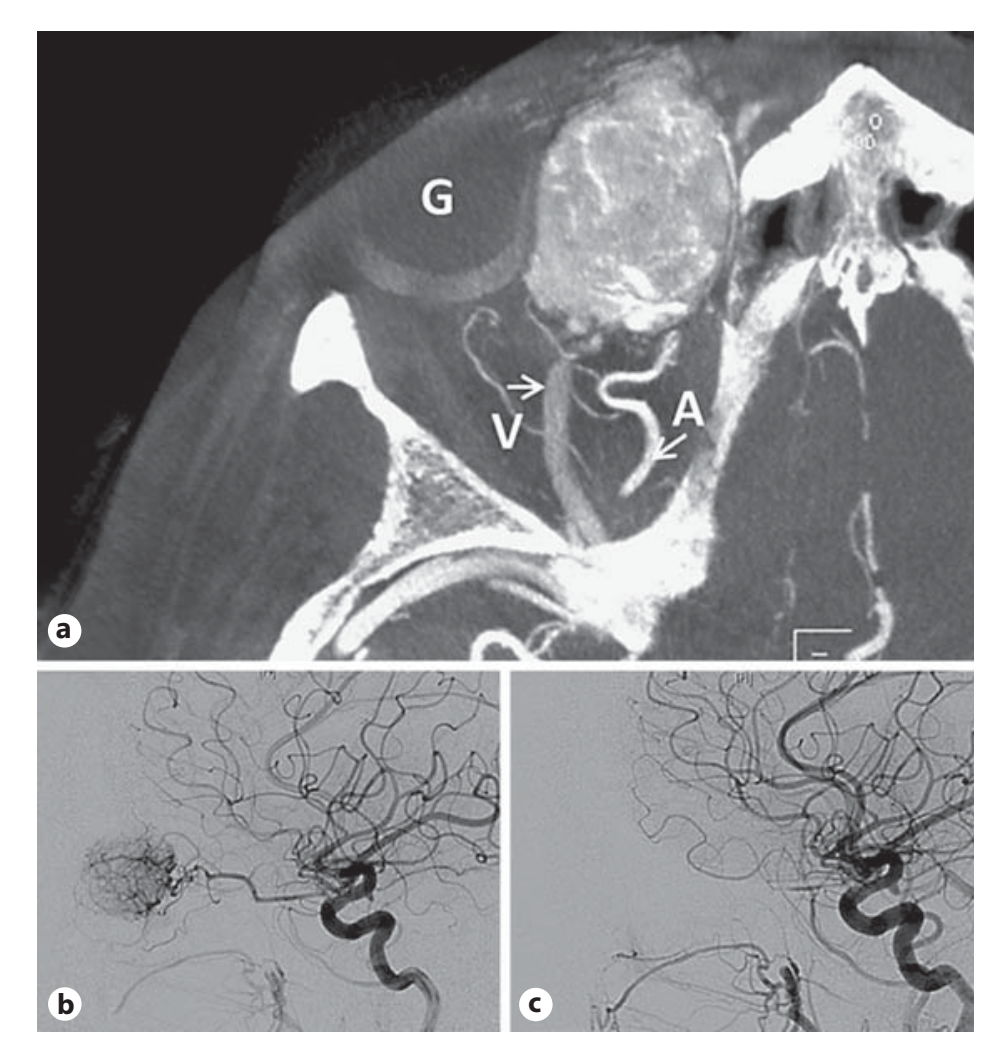

Fig. 1. Imaging of vascular tumor before and after embolization displacing the globe $(\mathrm{G})$ laterally and downward. a Axial CT with contrast examination showing a large hypervascular mass fed by long and short ciliary arteries from the right ophthalmic artery (A) in the region of the optic nerve. A large draining vein $(\mathrm{V})$ from the mass extended to the superior ophthalmic vein which then drained to the cavernous sinus. The vascular supply appeared very dysplastic and compatible with neovascularization. Pre- (b) and postembolization (c) angiography showing successful embolization with absence of tumor mass vascularization.

\section{Case Report}

A 55-year-old woman presented with right eye pain and proptosis, eyelid swelling, and abduction limitation progressive over several months. Orbital computed tomography, magnetic resonance imaging, and angiography demonstrated a well-circumscribed, highly vascular mass with multiple large flow voids lying adjacent to the optic nerve, displacing the globe laterally and downward, and supplied robustly from both long and short ciliary arteries off the right ophthalmic artery (fig. 1).

Due to the marked vascularity and high arterial flow within the mass, intra-arterial embolization of the ciliary feeding vessels using Onyx liquid embolic agent (Covidien, Mansfield, Mass., USA) was performed by an interventional neuroradiologist with postembolization imaging control showing complete obliteration of the feeding vessels (fig. 1). In short, a right femoral percutaneous approach was used with a Dyna computed tomography examination with $20 \%$ contrast injection into the right internal carotid artery to evaluate the supply to the orbital mass. Right internal carotid injection with filming over the orbit showed an extremely large hypervascular mass fed by long and short ciliary arteries from the right ophthalmic artery in the region of the optic nerve. A large draining vein from the mass extended to the superior ophthalmic vein, which then drained to the cavernous sinus. The vascular supply appeared very dysplastic and compatible with neovascularization. Right external carotid injection did not show any supply from the external carotid territory to the orbital mass. The orbital mass displaced the globe laterally and downward and had parasitized the ciliary arteries that would normally provide the choroidal blush, with several dysplastic vessels that extended to the supraorbital artery. The right ophthalmic artery was selectively catheterized, followed by devascularization of tumor supply utilizing aliquots of polyvinyl alcohol in a range of $45 \mu \mathrm{m}$ and followed by instillation of liquid Onyx at the entrance of the tumor vessels. Posttreatment right internal carotid injection showed complete devascularization of the tumor with stasis of contrast in the proximal ophthalmic artery. No choroidal blush was identified.

Embolization was followed immediately by orbitotomy and resection of a partially shrunken $2.7 \times 2 \times$ $1.5 \mathrm{~cm}$, irregularly shaped lesion with a glistening, micronodular, and hypervascular surface. Following surgical resection, right internal and right external carotid examinations revealed no choroidal blush. There has been complete surgical resection. 
Ocular Oncology

and Pathology

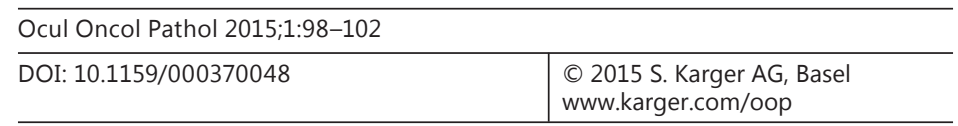

Hashemi et al.: Transarterial Onyx Embolization of an Orbital Solitary Fibrous Tumor
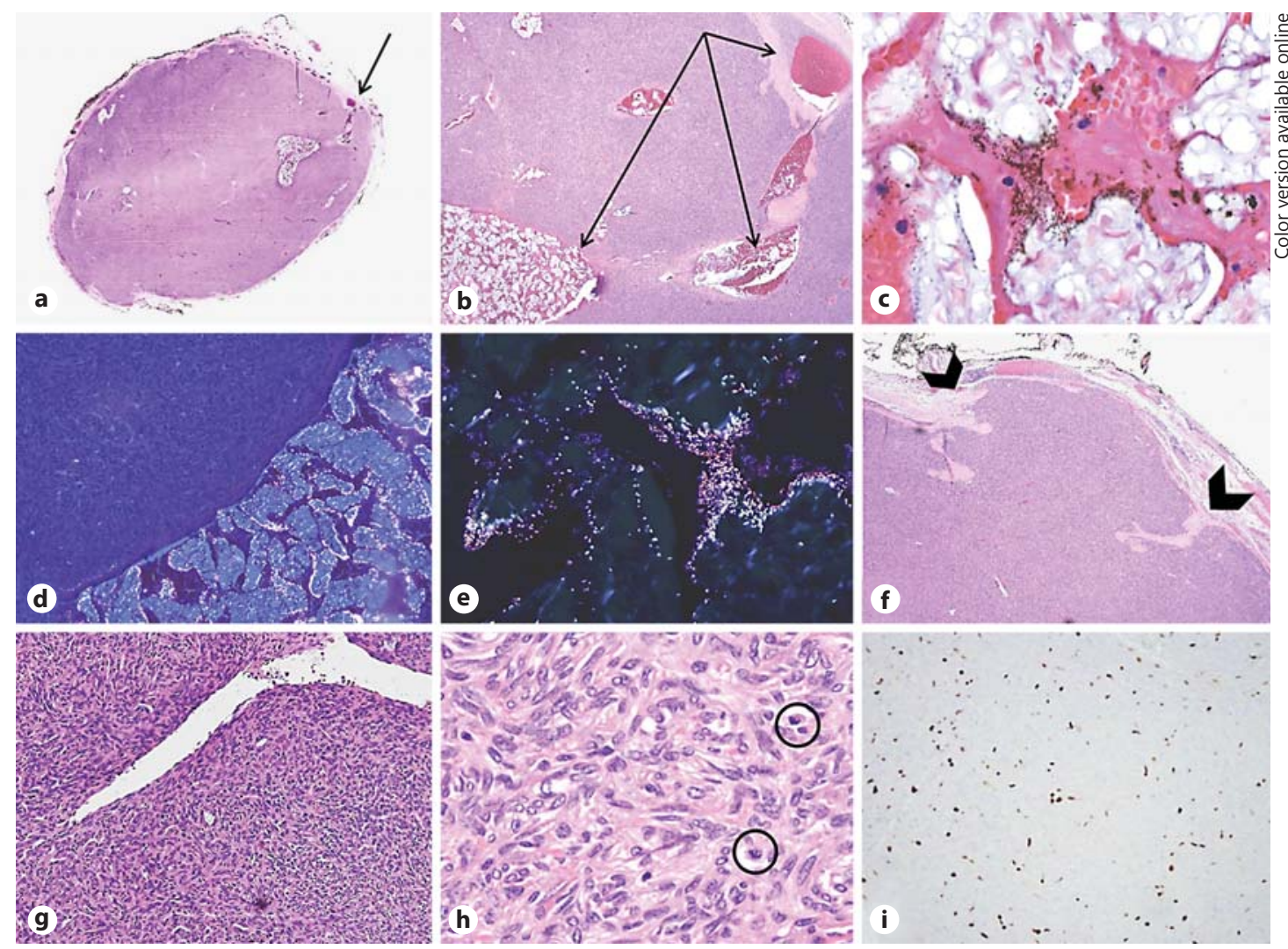

Fig. 2. Orbital tumor excised after embolization. a Low-power view of the large vessels entering the tumor (arrow). b Close-up view of the large vessels (arrows) partially containing the embolization material seen as white flaky particles admixed with blood. c High-power view of the embolization material in the lumen of the vessels. Note the two components: the granular black pigment (tantalum powder) and the white flaky-appearing material. $\mathbf{d}$ Low-power view of the tumor and the vessel containing the Onyx embolization material seen under polarization. Note the partially polarizable material at the center of the vessel. e High-power view of the intravascular material under polarization. Note that the granular black pigment is the component that polarizes and compare with $\mathbf{c}$. $\mathbf{f}$ Low-power view demonstrating the tumor infiltrating the fibrous capsule (between arrowheads). $\mathbf{g}$ Cellular tumor with angulated 'staghorn'-like vessels typically seen in tumors with hemangiopericytoma-like features. $\mathbf{h}$ High magnification of the tumor showing the spindle tumor cells with frequent mitoses (center of circles). $\mathbf{i}$ Increased proliferation rate is seen with Ki-67 marker. a-h Hematoxylin and eosin stain. $\mathbf{i}$ Immunohistochemistry, 3,3'-diaminobenzidine chromogen. Original magnification: $\times 1.25(\mathbf{a}), \times 4(\mathbf{b}), \times 20(\mathbf{c}, \mathbf{e}), \times 10(\mathbf{d}, \mathbf{g}, \mathbf{i}), \times 2(\mathbf{f}), \times 40(\mathbf{h})$.

Histopathology revealed a well-circumscribed, encapsulated neoplasm. There was a focal area of invasion and possible disruption of the capsule. The tumor was composed of patternless sheets of oval to spindled cells on a background of thin sinusoid and large 'staghorn'-type blood vessels with a MIB-1 (Ki-67) proliferation index of 5-10\%. Immunohistochemical stains demonstrated diffuse CD34 and BCL-2 positivity. Prominent vessels penetrating the fibrous capsule of the tumor were filled with the embolization material appearing as eosinophilic hyaline admixed with small polarizable granular black pigment (tantalum powder) [1]. The tumor adjacent to the embolized vessels showed no necrotic or inflammatory changes (fig. 2). These histopathologic findings were consistent with a diagnosis of orbital SFT with borderline malignant potential secondary to the increased proliferation index and cellularity immediately following feeder vessel embolization. Recommendation for close follow-up was made. 


\section{Comment}

Orbital SFT is a relatively uncommon spindle cell neoplasm most typically found in the superior orbit, where it presents with unilateral proptosis, progressive over months to years. Microscopically, SFT displays randomly oriented tumor cells without a defined pattern. Immunohistochemical stains demonstrate strong diffuse positivity to CD34, vimentin, CD99, and BCL-2 [2].

Historically, SFTs have been underdiagnosed, sometimes mistaken for hemangiopericytoma, fibrous histiocytoma, or giant cell angiofibroma [2]. Preoperative Onyx embolization has been reported for an array of vascular tumors of the head and neck to reduce hemorrhagic complications of surgery $[1,3]$. There is controversy over the classification of tumors that were previously designated as hemangiopericytoma and currently lumped into the category of SFT. One of the reasons the controversy exists is the type of vessels. In the typical description on hemangiopericytoma, there are the 'staghorn'- or 'anter'-like vessels associated with the mostly epithelioid cellular perivascular proliferation. However, these types of vessels are seen in most SFTs. There is now consensus that the vessels alone would not make the diagnosis of hemangiopericytoma more likely than the SFT. In the central nervous system, the diagnosis of hemangiopericytoma has prevailed over that of SFT because it is believed that this entity carries worse prognosis with more potential for malignant transformation. Since the orbit is a special site for the presence of these tumors, some have recommended separating those tumors that have more characteristic hemangiopericytoma histology from the more typical SFTs as they may be more frequently associated with malignant behavior [2].

Recently, vision-sparing preoperative Onyx embolization of orbital meningiomas fed by the ophthalmic artery has been described [4]. Onyx embolization of lesions may be performed transarterially or via direct percutaneous tumor puncture [3]. Transarterial embolization was used in this case due to the identification of posteriorly located main feeding vessels. Onyx liquid embolic agent is an ideal substance for embolizing orbital lesions of this type, because the slow precipitation properties of Onyx allows deep penetration into smaller vessels within the tumor, producing a diffuse infiltration and minimizing the need for repeated catheterizations $[3,4]$. The timing of the resection after embolization may be immediately after the radiologic interventional procedure or a few days following the embolization. To our knowledge, this is the first clinicopathologic report to include the histopathologic findings of Onyx in an orbital SFT in the English language literature. Although generally considered quite safe and without complication in the present case, rare potential complications of preoperative Onyx embolizations include skin and soft tissue necrosis, stroke, intracerebral hemorrhage, cranial nerve palsy, and death [5-7].

\section{Disclosure Statement}

The authors have no financial interest in any material discussed in this article.

\section{References}

1 Gemmete JJ, Chaudhary N, Pandey A, Gandhi D, Sullivan SE, Marentette LJ, Chepeha DB, Ansari SA: Usefulness of percutaneously injected ethylene-vinyl alcohol copolymer in conjunction with standard endovascular embolization techniques for preoperative devascularization of hypervascular head and neck tumors: technique, initial experience, and correlation with surgical observations. AJNR Am J Neuroradiol 2010;31:961966. 
-2 Furusato E, Valenzuela IA, Fanburg-Smith JC, Auerbach A, Furusato B, Cameron JD, Rushing EJ: Orbital solitary fibrous tumor: encompassing terminology for hemangiopericytoma, giant cell angiofibroma, and fibrous histiocytoma of the orbit: reappraisal of 41 cases. Hum Pathol 2011;42:120-128.

-3 Elhammady MS, Johnson JN, Peterson EC, Aziz-Sultan MA: Preoperative embolization of juvenile nasopharyngeal angiofibromas: transarterial versus direct tumoral puncture. World Neurosurg 2011;76:328-334.

-4 Trivelatto F, Nakiri GS, Manisor M, Riva R, Al-Khawaldeh M, Kessler I, Mounayer C: Preoperative onyx embolization of meningiomas fed by the ophthalmic artery: a case series. AJNR Am J Neuroradiol 2011;32:17621766.

5 Duffis EJ, Gandhi CD, Prestigiacomo CJ, Abruzzo T, Albuquerque F, Bulsara KR, et al: Head, neck, and brain tumor embolization guidelines. J Neurointerv Surg 2012;4:251-255.

-6 Wiegand S, Kureck I, Chapot R, Sesterhenn A, Bien S, Werner JA: Early side effects after embolization of a carotid body tumor using Onyx. J Vasc Surg 2010;52:742-745.

7 Ashour R, Aziz-Sultan MA, Soltanolkotabi M, Schoeneman SE, Alden TD, Hurley MC, Dipatri AJ, et al: Safety and efficacy of onyx embolization for pediatric cranial and spinal vascular lesions and tumors. Neurosurgery 2012; 71:773-784. 